\title{
Prevalence, Risk Factors and Antimicrobial Susceptibility of Beta Lactamase Producing Enterobacteriacea in a Tertiary Care Centre of Saudi Arabia
}

\author{
Gouse Basha Sheik ${ }^{1 *}$, Ahmed Sannat ${ }^{1}$, Bader Al-Rowis ${ }^{2}$, \\ Sahar M. Jeddawy ${ }^{2}$ and Doaa M.Sherif ${ }^{3}$ \\ ${ }^{1}$ Department of Medical Laboratory Sciences, College of Applied Medical Science's, \\ Al-Dawadmi, Shaqra University, Kingdom of Saudi Arabia \\ ${ }^{2}$ Al-Dawadmi General Hospital, Al- Dawadmi, Kingdom of Saudi Arabia \\ ${ }^{3}$ Mansoura Faculty of Medicine, Mansoura, Egypt \\ *Corresponding author
}

\section{A B S T R A C T}

\section{Keywords}

Beta lactamase, Antimicrobial resistance, Urinary tract infection, Enterobacteriacea

\begin{tabular}{l}
\hline Article Info \\
\hline Accepted: \\
11 January 2016 \\
Available Online: \\
11, February 2016
\end{tabular}

\section{Introduction}

Urinary tract infections (UTIs) along with
The main objective of this study was to determine the prevalence, risk factors and antimicrobial susceptibility of Beta lactamase (BL) producing Enterobacteriacea isolated from CAUTI and Non-CAUTI patients. This study was conducted at Dawadmi General Hospital, Saudi Arabia from March 2012 to March 2015. A total of 780 urine samples were collected from mid-stream, clean catch, suprapubic aspirate and Foley's catheter from both inpatient and outpatient departments for studying the presence of Beta lactamase enzymes producing Enterobacteriacea. The organisms were isolated by standard microbiological techniques and the isolates were tested for their species identification and antimicrobial susceptibility by modified Vitek 2 method. Out of the total 780 urine samples collected in this study, $388(49.7 \%)$ came out to be positive for UTI isolates. Among them 231 (29.6\%) isolates recovered were found to be Enterobacteriacea. Among Enterobacteriacea $150(64.9 \%)$ isolates were producing beta lactamases and $81(35.1 \%)$ isolates were non-beta lactamases. The risk factors in Beta lactamase isolates were found to be long term hospital stay $134(89.3 \%)$, urinary catheter $125(83.3 \%)$, ICU stay $109(72.7 \%)$, prior antibiotic use within 3 months $98(65.3 \%)$, obstructive uropathy 74(46.7\%), renal disease/renal transplant 68(45.3\%). The frequency of ESBL, CTX$\mathrm{M}, \mathrm{AmpC}$, ESBL with Carbapenems, OXA and MBL were higher in catheterized patients as compared to non-catheterized patients. The $\beta$-lactamases producing isolates were resistant to most of the antibiotics tested but Tigecycline and Colistin showed promising efficacy against all the Beta lactamases in both CAUTI patients and Non-CAUTI patients Catheterized patients should be screened regularly for the prevalence beta lactamase producers. While handling catheterized patients the healthcare staff should follow proper guidelines and infection control measures to avoid the spread of nosocomial infections. 
common type of healthcare-associated infection, secondly to Urinary system infections(USI) which accounts for more than $15 \%$ of infections as reported by acute care hospitals(Magill et al., 2012). Among Enterobacteriaceae particularly Escherichia coli and Klebsiella pneumoniae are considered as the major causes of UTI in both inpatients and outpatients (Sharma et al., 2012). Since the aetiology of UTI and the antibiotic susceptibility pattern of urinary pathogens have been changing over the past years, current knowledge on the burden and antimicrobial susceptibility pattern is essential for appropriate therapy. Gram negative bacteria has more than 1000 Beta lactamase(BL) producers which include Extended spectrum Beta lactamases (ESBLs), AmpC -lactamases (AmpC), Klebsiella pneumoniae carbapenemases (KPC), and the metallo - lactamases (MBLs) which are multidrug resistant bacteria. (Bush, 2011) They may also produce multiple Beta Lactamases such as combinations of ESBLs and carbapenemases, ESBLs and AmpC etc., The most common pathogens involved in producing beta lactamases are Escherichia coli and Klebsiella pneumoniae which produce the enzymes Extended Spectrum $\beta$ Lactamases (ESBLs). Catheter-associated urinary tract infections (CAUTI) accounts for $30 \%$ to $40 \%$ among all the nosocomial infections (Conway et al., 2012). CAUTI occur in patients having urinary tract infections on the third day after insertion of catheter. In long term catheter use, the risk of antibiotic resistant pathogens further increases due to the formation of bio-film along the catheter. (Conway et al., 2012). The risk of infection in patients catheterized for more than 7 days is $25 \%$ and increases to 100\% after 30 days (Jaggi et al, 2012). The colonization of bacteria the drainage bags of catheterized patients is found to be main source of outbreaks of resistant bacteria in acute care facilities (Hooton et al., 2009, Lo et al., 2014). The main objective of this study was to study was to determine the prevalence and antimicrobial susceptibility of Beta lactamase (BL) producing Enterobacteriacea isolated from CAUTI and Non-CAUTI patients in Al-Dawadmi General Hospital, Saudi Arabia This study is important for clinicians in order to facilitate the effective treatment and management of patient with symptoms of urinary tract infections.

\section{Materials and Methods}

This retrospective study was conducted at the Department of Medical Microbiology, Al-Dawadmi General Hospital, AlDawadmi Governorate, Kingdom of Saudi Arabia, KSA, a tertiary care 250 bed capacity hospital, with a CBAHI (Central Board for Accreditation of Health Institutes) accredited Lab, from March 2012 till March 2015. A total of 780 urine samples were collected from patient with symptoms suggestive UTI from both in and out patients using systematic random sampling technique. Socio-demographic characteristics such as gender and age were gathered from eligible patients. Clinical features such as history of hospitalization, ICU admission, prior antibiotic use within 3 months, long term hospital stay, presence of urinary catheter and chronic diseases were also collected. Urinary samples included mid-stream clean catch urine and urine obtained from Foley's catheter from both in and out patients. Urine samples were cultured using a $1 \mu \mathrm{m}$ calibrated loop onto CLED agar and blood agar (Media, Ministry of Health), incubated at $37^{\circ} \mathrm{C}$ for $18-24$ hours and the number of colonies was counted. The specimen yielding more than or equal to $10^{5} \mathrm{CFU} / \mathrm{ml}$ of urine was interpreted as significant, except for samples from elderly women and men. Colony count 
of $10^{4}$ and $10^{3} \mathrm{CFU} / \mathrm{ml}$ of urine respectively was interpreted as significant. Plates with more than 3 different types of organisms were considered mixed insignificant growth, all the plates were inspected for growth and the isolates were identified by observing colony morphology, Gram-stain characteristics and relevant biochemical tests by Vitek 2 system for microbial identification and sensitivity Instrument. The isolates were tested for their antimicrobial susceptibility using AST N291 and N-232. Reference strains included in the study were as follows: ESBL positive Klebsiella pneumoniae ATCC 700603, ESBL negative Escherichia coli ATCC 25922. The results were interpreted according to the guidelines of Clinical and Laboratory Standards Institute (CLSI, 2012).

\section{Statistical Analysis}

Statistical analyses were performed using the SPSS, Version 20. Chi-square test was used to compare categorical variables were ever applicable. A p-value less than 0.05 were considered significant.

\section{Results and Discussion}

Out of the total 780 urine samples collected in this study, $388(49.7 \%)$ came out to be positive for UTI isolates. Among them 231 $(29.6 \%)$ isolates recovered were found to be Enterobacteriacea. Among Enterobacteriaceae $150(64.9 \%)$ isolates were producing beta lactamases and $81(35.1 \%)$ isolates were non-beta lactamases. The overall prevalence of beta lactamases producing Enterobacteriacea in males was 36(24\%) which are found to be less than the females $114(76 \%)$ which was statistically significant $(p<0.05)$. Among inpatients the majority of beta lactamases producing Enterobacteriacea are from patients in Intensive care unit $30(30.3 \%)$ followed by Medical ward
26(26.3\%) Surgical ward 23(23.2\%), Paediatric ward 11(11.1\%) and the least were from Obstetrics and gynaecology $9(9.1 \%)$ which was statistically significant $(\mathrm{p}<0.05)$. Most of beta lactamases producing Enterobacteriacea infected patients belonged to the 31-45 years age group 46(30.7\%) followed by $>62$ years $39(26 \%)$ and $16-30$ years $32(21.3 \%)$ respectively which was statistically significant $(p<0.05)$. Similarly the percentage of beta lactamases producing Enterobacteriacea in outpatients was $51(34 \%)$ which are less than the inpatients $99(66 \%)$ which was statistically significant $(\mathrm{p}<0.05)$. The percentage of beta lactamases (BL) producers is more $90(60 \%)$ in catheter associated UTI patients (CAUTI) when compared to non-catheter associated UTI patients (Non-CAUTI) which was only $60(40 \%)$ which was statistically significant $(p<0.05)$. The results were shown in Table 1 .

Escherichia coli was the most predominant one having 66(44\%) isolates of beta lactamases producing Enterobacteriacea followed by Klebsiella spp 46(30.7\%), Enterobacter species 15(10\%), Proteus mirabilis 10(6.7\%), Acinetobacter species $7(4.7 \%)$, Serratia species $4(2.7 \%)$ and Citrobacter species 2(1.3\%). Among $90 \mathrm{BL}$ isolates from CAUTI patients, 56(62.2\%) were ESBL, 5(5.6\%) were CTX-M, 11(12.2\%) were AmpC, 11(12.2\%) were ESBL with Carbapenems, 3(3.3\%) were OXA and $4(4.4 \%)$ were MBL producing isolates as shown in Figure.1. Among $60 \mathrm{BL}$ isolates from Non - CAUTI patients, $29(48 \%)$ were ESBL, 4(2.7\%) were CTX$\mathrm{M}, 9(6 \%)$ were AmpC, 13(8.7\%) were ESBL with Carbapenems, 2(1.3\%) were OXA, 3(2\%) were MBL producing isolates as shown in Figure.2. The results showed that the BL isolates in catheter associated UTI patients (CAUTI) were more 90(60\%) when compared to non-catheter associated 
UTI patients (Non-CAUTI) which was only $60(40 \%)$ as shown in Table 2. Risk factors associated with BL UTI were analyzed by comparing patients with non-BL UTIs. The risk factors in Beta lactamase isolates were found to be long term hospital stay $134(89.3 \%)$, urinary catheter $125(83.3 \%)$, ICU stay $109(72.7 \%)$, prior antibiotic use within 3 months 98(65.3\%), obstructive uropathy $74(46.7 \%)$, renal disease/renal transplant $68(45.3 \%)$. The risk factors in non-BL UTI is less when comparing to BL UTI as shown in the Table 3.

Rates of antibiotic resistance for beta lactamases producing Enterobacteriacea in CAUTI patients and Non-CAUTI patients are summarized in Table 4. Antibiotic resistance rates for CAUTI patients were greater than Non-CAUTI patients. ESBL isolates showed high rate of resistance to Ampicillin, Ceftazidime, Cifotaxime and Trimethoprim sulfamethoxazole in both CAUTI patients and Non-CAUTI patients. ESBL and CTX-M isolates showed high rate of resistance to Cefipime in addition to Ampicillin, Ceftazidime, Cifotaxime and Trimethoprim sulfamethoxazole in both CAUTI patients and Non-CAUTI patients. AmpC isolates showed high rate of resistance to even amoxicillin clavunalic acid, Cefoxitin in addition to Ampicillin, Ceftazidime, Cifotaxime and Trimethoprim sulfamethoxazole in both CAUTI patients and Non-CAUTI patients. ESBL and Carbapenem isolates showed high rate of resistance to Imipenem and Meropenem in addition to Ampicillin, Ceftazidime, Cifotaxime and Trimethoprim sulfamethoxazole in both CAUTI patients and Non-CAUTI patients. OXA isolates showed high rate of resistance to amoxicillin clavunalic acid, Imipenem and Meropenem, Amikacin, Gentamicin addition to Ampicillin, Ceftazidime, Cifotaxime and Trimethoprim sulfamethoxazole in both
CAUTI patients and Non-CAUTI patients. MBL isolates showed high rate of resistance to amoxicillin clavunalic acid, Imipenem and Meropenem Norfloxacin in addition to Ampicillin, Ceftazidime, Cifotaxime and Trimethoprim sulfamethoxazole in both CAUTI patients and Non-CAUTI patients.

The current study aimed to study prevalence of antibiotic resistance enzymes among Enterobacteriacea isolated from different cases of urinary tract infections in AlDawadmi General Hospital, Saudi Arabia and also to analyze the antimicrobial sensitivity pattern of those isolates against various types of antimicrobial agents used for treating urinary tract infections. Previous studies conducted on Beta Lactamase resistance of urinary tract infections in hospital and community patients not focused on CAUTI and non-CAUTI patients (Zilberberg et al., 2013; Azap et al., 2010; Calbo et al., 2006). Since CAUTI patients have different antibiotic resistance patterns those of non-CAUTI patients study of prevalence rate, antimicrobial resistance pattern and risk factors for beta lactamase producers unique to this population is important for the development of effective preventive measures (Yamamichi et al., 2012). In our study various $\beta$ lactamases were found both in CAUTI and non-CAUTI patients but the number is more in CAUTI than the non-CAUTI patients. Previous studies showed that patients having chronic indwelling catheters acting as the most common site of isolation of resistant gram negative organisms (Arnaldo et al., 2013, Mody et al., 2007). Among the $\beta$ lactamases ESBL production was found to be high $(56.7 \%)$ in both CAUTI and Non-CAUTI which is higher than reports on ESBL production by Enterobacteriaceae from Saudi Arabia which reported (35.16\%, $39.8 \%, 35 \%, 27.5 \%)$ by Loveena et al (2013); Bandekar et al (2011); Babay 
(2002); Bilal and Gedebou, (2002). However higher rates of ESBL production were reported by Agamy et al (2009) (55\%) from Saudi Arabia, and by Mathur et al (2002)(68\%) from India. and Pakistan Shah et al (2002)(45\%).

The overall prevalence of beta lactamases producing Enterobacteriacea in males was $36(24 \%)$ which are found to be less than the females $114(76 \%)$. In a study conducted by Shafkat qamar (2015) in Saudi Arabia showed that among BL isolates from Enterobacteriacea 135 (39\%) were male and $210(61 \%)$ were female patients. In our study, $66 \%$ of the BL producing Enterobacteriacea isolates are from inpatients which is differs from other studies that reported high prevalence of BL in outpatients (Hernandez et al., 2003; Mendonca et al., 2007). Among inpatients the majority of beta lactamases producing Enterobacteriacea are from patients in Intensive care unit 109(72.7\%).This coincides with the study conducted by AbdulRahman et al (2004) who found that the majority of ESBL isolates were from patients under long term care followed by ICU $(80.9 \%)$. In our study, Most of beta lactamases producing Enterobacteriacea infected belonged to the 31-45 years age group $46(30.7 \%)$ followed by > 62 years $39(26 \%)$ and $16-30$ years $32(21.3 \%)$ respectively.Our study showed that Escherichia coli was significantly the most predominant one representating 66(44\%) of isolates of beta lactamases producing Enterobacteriacea followed by Klebsiella spp 46(30.7\%). This result coincides with the previous studies conducted by Karou $e t$ al (2009) and Moyo et al (2010).

Multi type $\beta$-lactamases producing isolates were also reported in our study. Among beta lactamases producing Enterobacteriacea 85(56.7\%) were ESBL, 9(6\%) were CTX-
M, 20(13.3\%) were AmpC, 24(16\%) were ESBL with Carbapenems, 5(3.3\%) were OXA and 7(4.7\%) were MBL producing isolates. In a similar study, Amreliwala et al (2015) reported that $52.5 \%$ of the isolates included in the study were ESBL producers, 25.25\% were ESBL + AmpC co-producers and $22.25 \%$ were only AmpC producers. In a study conducted by Loveena et al (2013) of the 273 gram negative isolates, 96(35.16\%) were ESBL producers, followed by $30(10.98 \%)$ metallo $\beta$-lactamase (MBL) producers and 15(5.4\%) AmpC producers. In a study conducted by Khalid et al (2010) in Saudi Arabia indicate that $71 \%$ of ESBL isolates contain CTX-M genes. In our study antibiotic resistance rates for CAUTI patients were significantly greater than NonCAUTI patients. Both BL and non-BL isolates were more resistant to antibiotics typically recommended for empirical initial therapy for UTI such as Ampicillin and Trimethoprim-sulphamethoxazole, whereas $\mathrm{BL}$ isolates were often resistant to antibiotics including Ceftazidime, Cifotaxime, Cefoxitin, Amoxicillin Clavunalic acid and Carbapenems in both CAUTI patients and Non-CAUTI patients.

In our study, ESBL isolates showed high rate of resistance to Ampicillin, Ceftazidime, Cifotaxime and Trimethoprim sulfamethoxazole in both CAUTI patients and Non-CAUTI patients. Susceptibility pattern of ESBL-producing isolates showed that these strains are not only resistant to beta-lactams but also to other classes of antibacterials including Gentamicin and Ciprofloxacin in both CAUTI patients and Non-CAUTI patients. The only beta-lactams which were active against the CTX-M, AmpC and the ESBL co-producers were the Carbapenems such as Imipenem and Meropenem in both CAUTI patients and Non-CAUTI patients. 
Table.1 Characteristics of UTI Patients Caused by BL Isolates at Al-Dawadmi General Hospital

\begin{tabular}{|c|c|c|c|}
\hline Characteristics & $\begin{array}{c}\text { Total } \\
\text { Enterobacteriacea }(\mathrm{N}=\mathbf{2 3 1})\end{array}$ & $\begin{array}{c}\text { BL } \\
(\mathrm{N}=150)\end{array}$ & P-value \\
\hline Male & $82(35.5 \%)$ & $36(24 \%)$ & \\
\hline Female & $149(64.5 \%)$ & $114(76 \%)$ & $<0.05$ \\
\hline $\begin{array}{c}\text { Age(Years) } \\
\leq 15\end{array}$ & $21(9.1 \%)$ & $9(6 \%)$ & \multirow{5}{*}{$<0.05$} \\
\hline $16-30$ & $46(20 \%)$ & $32(21.3 \%)$ & \\
\hline $31-45$ & $68(29.4 \%)$ & $46(30.7 \%)$ & \\
\hline $46-60$ & $34(14.7 \%)$ & $24(16 \%)$ & \\
\hline$\geq 61$ & $62(26.8 \%)$ & $39(26 \%)$ & \\
\hline Outpatient & $87(37.7 \%)$ & $51(34 \%)$ & \multirow[b]{2}{*}{$<0.05$} \\
\hline Inpatient & $144(62.3 \%)$ & $99(66 \%)$ & \\
\hline Medical ward & $43(43.8 \%)$ & $26(26.3 \%)$ & \multirow{5}{*}{$<0.05$} \\
\hline Surgery ward & $29(20.1 \%)$ & $23(23.2 \%)$ & \\
\hline Pediatric ward & $23(16 \%)$ & $11(11.1 \%)$ & \\
\hline Intensive care unit & $36(11.1 \%)$ & $30(30.3 \%)$ & \\
\hline Obstetrics and gynecology & $13(9 \%)$ & $9(9.1 \%)$ & \\
\hline CAUTI & $121(52.4 \%)$ & $90(60 \%)$ & \multirow[b]{2}{*}{$<0.05$} \\
\hline NON-CAUTI & $110(47.6 \%)$ & $6040 \%)$ & \\
\hline
\end{tabular}

BL-Beta Lactamases producing isolates 
Table.2 BL Isolates in CAUTI and Non-CAUTI Patients

\begin{tabular}{|c|c|c|c|c|c|c|c|c|c|c|c|c|}
\hline \multirow{3}{*}{ Organism } & \multicolumn{12}{|c|}{ Number and \% of BL isolates $(\mathrm{N}=150)$} \\
\hline & \multicolumn{2}{|c|}{$\begin{array}{c}\text { ESBL } \\
(\mathbf{N}=\mathbf{8 5})\end{array}$} & \multicolumn{2}{|c|}{$\begin{array}{c}\text { ESBL + } \\
\text { CTX - M } \\
(\mathbf{N}=09)\end{array}$} & \multicolumn{2}{|c|}{$\begin{array}{c}\text { AmpC } \\
(\mathbf{N}=\mathbf{2 0})\end{array}$} & \multicolumn{2}{|c|}{$\begin{array}{c}\text { Carbapenems + } \\
\text { ESBL } \\
(\mathbf{N}=\mathbf{2 4})\end{array}$} & \multicolumn{2}{|c|}{$\begin{array}{c}\text { OXA } \\
(N=05)\end{array}$} & \multicolumn{2}{|c|}{$\begin{array}{c}\text { MBL } \\
(\mathbf{N}=\mathbf{0 7})\end{array}$} \\
\hline & CAUTI & $\begin{array}{c}\text { Non- } \\
\text { CAUTI }\end{array}$ & CAUTI & $\begin{array}{c}\text { Non- } \\
\text { CAUTI }\end{array}$ & CAUTI & $\begin{array}{c}\text { Non- } \\
\text { CAUTI }\end{array}$ & CAUTI & $\begin{array}{c}\text { Non- } \\
\text { CAUTI }\end{array}$ & CAUTI & $\begin{array}{c}\text { Non- } \\
\text { CAUTI }\end{array}$ & CAUTI & $\begin{array}{c}\text { Non- } \\
\text { CAUTI }\end{array}$ \\
\hline $\begin{array}{c}E . \text { coli } \\
(N=66)\end{array}$ & 25 & 12 & 05 & 04 & 08 & 07 & 03 & 02 & - & - & - & - \\
\hline $\begin{array}{c}\text { Klebsiella } \\
\text { pneumonia } \\
(N=46)\end{array}$ & 22 & 13 & - & - & - & - & 05 & 06 & - & - & - & - \\
\hline $\begin{array}{c}\text { Enterobacter } \\
\text { species } \\
(N=15)\end{array}$ & 05 & 03 & - & - & 03 & - & 02 & 02 & - & - & - & - \\
\hline $\begin{array}{c}\text { Proteus } \\
\text { mirabilis } \\
(N=10)\end{array}$ & 04 & 01 & - & - & - & - & 01 & 03 & - & - & - & 01 \\
\hline $\begin{array}{c}\text { Acinetobacter } \\
\text { species } \\
(N=7)\end{array}$ & - & - & - & - & - & - & - & - & 03 & 02 & 01 & 01 \\
\hline $\begin{array}{c}\text { Citrobacter } \\
\text { Species } \\
(N=02)\end{array}$ & - & - & - & - & - & 02 & - & - & - & - & - & - \\
\hline $\begin{array}{c}\text { Serratia species } \\
\quad(N=04)\end{array}$ & - & - & - & - & - & - & - & - & - & - & 03 & 01 \\
\hline $\begin{array}{c}\text { Total } \\
(N=231)\end{array}$ & 56 & 29 & 05 & 04 & 11 & 09 & 11 & 13 & 03 & 02 & 04 & 03 \\
\hline
\end{tabular}

CAUTI-Catheter Associated UTI; Non-CAUTI- Non-Catheter Associated UTI 
Table.3 Resistance Patterns of BL Isolates among CAUTI and Non-CAUTI Patients

\begin{tabular}{|c|c|c|c|c|c|c|c|c|c|c|c|c|}
\hline \multirow[t]{3}{*}{ Antibacterial agent } & \multicolumn{12}{|c|}{ Percentage of Resistance of $\mathrm{BL}$ isolates $(\mathrm{N}=150)$} \\
\hline & \multicolumn{2}{|c|}{$\begin{array}{c}\text { ESBL } \\
(\mathbf{N}=\mathbf{8 5})\end{array}$} & \multicolumn{2}{|c|}{$\begin{array}{c}\text { ESBL }+ \\
\text { CTX - M }\end{array}$} & \multicolumn{2}{|c|}{$\begin{array}{c}\text { AmpC } \\
(\mathbf{N}=\mathbf{2 0})\end{array}$} & \multicolumn{2}{|c|}{$\begin{array}{c}\text { Carbapenems + ESBL } \\
(\mathrm{N}=24)\end{array}$} & \multicolumn{2}{|c|}{$\begin{array}{c}\text { OXA } \\
(\mathbf{N}=05)\end{array}$} & \multicolumn{2}{|c|}{$\begin{array}{c}\text { MBL } \\
(\mathbf{N}=\mathbf{0 7})\end{array}$} \\
\hline & CAUTI & $\begin{array}{c}\text { Non- } \\
\text { CAUTI }\end{array}$ & CAUTI & $\begin{array}{c}\text { Non- } \\
\text { CAUTI }\end{array}$ & CAUTI & $\begin{array}{c}\text { Non- } \\
\text { CAUTI }\end{array}$ & CAUTI & $\begin{array}{c}\text { Non- } \\
\text { CAUTI }\end{array}$ & CAUTI & $\begin{array}{c}\text { Non- } \\
\text { CAUTI }\end{array}$ & CAUTI & Non-CAUTI \\
\hline Ampicillin & $\begin{array}{c}56 \\
(100)\end{array}$ & $\begin{array}{c}27 \\
(93.1)\end{array}$ & $\begin{array}{c}5 \\
(100)\end{array}$ & $\begin{array}{c}4 \\
(100)\end{array}$ & $\begin{array}{c}11 \\
(100)\end{array}$ & $\begin{array}{c}9 \\
(100)\end{array}$ & $\begin{array}{c}11 \\
(100)\end{array}$ & $\begin{array}{c}12 \\
(92.3)\end{array}$ & $\begin{array}{c}3 \\
(100)\end{array}$ & $\begin{array}{c}2 \\
(100)\end{array}$ & $\begin{array}{c}4 \\
(100)\end{array}$ & $\begin{array}{c}3 \\
(100)\end{array}$ \\
\hline $\begin{array}{c}\text { Amoxycillin-Clavunalic } \\
\text { acid }\end{array}$ & $\begin{array}{c}40 \\
(71.4)\end{array}$ & $\begin{array}{c}16 \\
(55.2)\end{array}$ & $\begin{array}{c}2 \\
(40)\end{array}$ & $\begin{array}{c}1 \\
(25)\end{array}$ & $\begin{array}{c}11 \\
(100)\end{array}$ & $\begin{array}{c}7 \\
(77.8)\end{array}$ & $\begin{array}{c}11 \\
(100)\end{array}$ & $\begin{array}{c}11 \\
(84.6)\end{array}$ & $\begin{array}{c}3 \\
(100)\end{array}$ & $\begin{array}{c}2 \\
(100)\end{array}$ & $\begin{array}{c}4 \\
(100)\end{array}$ & $\begin{array}{c}3 \\
(100)\end{array}$ \\
\hline Ceftazidime & $\begin{array}{c}51 \\
(91.1)\end{array}$ & $\begin{array}{c}23 \\
(79.3)\end{array}$ & $\begin{array}{c}5 \\
(100)\end{array}$ & $\begin{array}{c}4 \\
(100)\end{array}$ & $\begin{array}{c}11 \\
(100)\end{array}$ & $\begin{array}{c}9 \\
(100)\end{array}$ & $\begin{array}{c}11 \\
(100)\end{array}$ & $\begin{array}{c}10 \\
(76.9)\end{array}$ & $\begin{array}{c}3 \\
(100)\end{array}$ & $\begin{array}{c}2 \\
(100)\end{array}$ & $\begin{array}{c}4 \\
(100)\end{array}$ & $\begin{array}{c}3 \\
(100)\end{array}$ \\
\hline Cifotaxime & $\begin{array}{c}56 \\
(100)\end{array}$ & $\begin{array}{c}24 \\
(82.8)\end{array}$ & $\begin{array}{c}5 \\
(100)\end{array}$ & $\begin{array}{c}4 \\
(100)\end{array}$ & $\begin{array}{c}11 \\
(100)\end{array}$ & $\begin{array}{c}8 \\
(88.9)\end{array}$ & $\begin{array}{c}10 \\
(90.9)\end{array}$ & $\begin{array}{c}11 \\
(84.6)\end{array}$ & $\begin{array}{c}3 \\
(100)\end{array}$ & $\begin{array}{c}2 \\
(100)\end{array}$ & $\begin{array}{c}4 \\
(100)\end{array}$ & $\begin{array}{c}3 \\
(100)\end{array}$ \\
\hline Cefoxitin & $\begin{array}{c}16 \\
(28.6)\end{array}$ & $\begin{array}{c}2 \\
(6.9)\end{array}$ & $\begin{array}{c}5 \\
(100)\end{array}$ & $\begin{array}{c}4 \\
(100)\end{array}$ & $\begin{array}{c}10 \\
(90.9)\end{array}$ & $\begin{array}{c}7 \\
(77.8)\end{array}$ & $\begin{array}{c}6 \\
(54.5)\end{array}$ & $\begin{array}{c}4 \\
(30.8)\end{array}$ & $\begin{array}{c}2 \\
(66.7)\end{array}$ & 0 & $\begin{array}{c}4 \\
(100)\end{array}$ & $\begin{array}{c}2 \\
(66.7)\end{array}$ \\
\hline Cefipime & $\begin{array}{c}10 \\
(17.9)\end{array}$ & $\begin{array}{c}4 \\
(13.8)\end{array}$ & $\begin{array}{c}5 \\
(100)\end{array}$ & $\begin{array}{c}4 \\
(100)\end{array}$ & $\begin{array}{c}5 \\
(45.5)\end{array}$ & $\begin{array}{c}3 \\
(33.3)\end{array}$ & $\begin{array}{c}3 \\
(27.3)\end{array}$ & $\begin{array}{c}3 \\
(23.1)\end{array}$ & $\begin{array}{c}1 \\
(33.3)\end{array}$ & $\begin{array}{c}1 \\
(50)\end{array}$ & $\begin{array}{c}2 \\
(50)\end{array}$ & $\begin{array}{c}2 \\
(66.7)\end{array}$ \\
\hline Imipenem & $\begin{array}{c}4 \\
(7.1)\end{array}$ & $\begin{array}{c}0 \\
(0)\end{array}$ & $\begin{array}{c}0 \\
(0)\end{array}$ & $\begin{array}{c}0 \\
(0)\end{array}$ & $\begin{array}{c}2 \\
(18.2)\end{array}$ & $\begin{array}{c}0 \\
(0)\end{array}$ & $\begin{array}{c}11 \\
(100)\end{array}$ & $\begin{array}{c}9 \\
(69.2)\end{array}$ & $\begin{array}{c}3 \\
(100)\end{array}$ & $\begin{array}{c}2 \\
(100)\end{array}$ & $\begin{array}{c}4 \\
(100)\end{array}$ & $\begin{array}{c}3 \\
(100)\end{array}$ \\
\hline Meropenem & $\begin{array}{c}3 \\
(5.4)\end{array}$ & $\begin{array}{c}0 \\
(0)\end{array}$ & $\begin{array}{c}0 \\
(0)\end{array}$ & $\begin{array}{c}0 \\
(0)\end{array}$ & $\begin{array}{c}2 \\
(18.2)\end{array}$ & $\begin{array}{c}1 \\
(11.1)\end{array}$ & $\begin{array}{c}10 \\
(90.9)\end{array}$ & $\begin{array}{c}11 \\
(84.6)\end{array}$ & $\begin{array}{c}3 \\
(100)\end{array}$ & $\begin{array}{c}2 \\
(100)\end{array}$ & $\begin{array}{c}4 \\
(100)\end{array}$ & $\begin{array}{c}3 \\
(100)\end{array}$ \\
\hline Amikacin & $\begin{array}{c}23 \\
(41.1)\end{array}$ & $\begin{array}{c}10 \\
(34.5)\end{array}$ & $\begin{array}{c}3 \\
(60)\end{array}$ & $\begin{array}{c}2 \\
(50)\end{array}$ & $\begin{array}{c}4 \\
(36.4)\end{array}$ & $\begin{array}{c}2 \\
(22.2)\end{array}$ & $\begin{array}{c}7 \\
(63.6)\end{array}$ & $\begin{array}{c}5 \\
(38.5)\end{array}$ & $\begin{array}{c}3 \\
(100)\end{array}$ & $\begin{array}{c}2 \\
(100)\end{array}$ & $\begin{array}{c}3 \\
(75)\end{array}$ & $\begin{array}{c}2 \\
(66.7)\end{array}$ \\
\hline Gentamicin & $\begin{array}{c}29 \\
(51.8)\end{array}$ & $\begin{array}{c}15 \\
(51.7)\end{array}$ & $\begin{array}{c}3 \\
(60)\end{array}$ & $\begin{array}{c}3 \\
(75)\end{array}$ & $\begin{array}{c}4 \\
(36.4)\end{array}$ & $\begin{array}{c}3 \\
(33.3)\end{array}$ & $\begin{array}{c}3 \\
(27.3)\end{array}$ & $\begin{array}{c}4 \\
(30.8)\end{array}$ & $\begin{array}{c}3 \\
(100)\end{array}$ & $\begin{array}{c}2 \\
(100)\end{array}$ & $\begin{array}{c}1 \\
(25)\end{array}$ & $\begin{array}{c}1 \\
(33.3)\end{array}$ \\
\hline Ciprofloxacin & $\begin{array}{c}30 \\
(53.6)\end{array}$ & $\begin{array}{c}19 \\
(65.5)\end{array}$ & $\begin{array}{c}3 \\
(60)\end{array}$ & $\begin{array}{c}2 \\
(50)\end{array}$ & $\begin{array}{c}6 \\
(54.5)\end{array}$ & $\begin{array}{c}4 \\
(44.4)\end{array}$ & $\begin{array}{c}4 \\
(36.4)\end{array}$ & $\begin{array}{c}5 \\
(38.5)\end{array}$ & $\begin{array}{c}1 \\
(33.3)\end{array}$ & $\begin{array}{c}1 \\
(50)\end{array}$ & $\begin{array}{c}2 \\
(50)\end{array}$ & $\begin{array}{c}1 \\
(33.3)\end{array}$ \\
\hline Norfloxacin & $\begin{array}{c}17 \\
(30.4)\end{array}$ & $\begin{array}{c}10 \\
(34.5)\end{array}$ & $\begin{array}{c}2 \\
(40)\end{array}$ & $\begin{array}{c}2 \\
(50)\end{array}$ & $\begin{array}{c}5 \\
(45.5)\end{array}$ & $\begin{array}{c}4 \\
(44.4)\end{array}$ & $\begin{array}{c}8 \\
(72.7)\end{array}$ & $\begin{array}{c}6 \\
(46.2)\end{array}$ & $\begin{array}{c}2 \\
(66.7)\end{array}$ & $\begin{array}{c}1 \\
(50)\end{array}$ & $\begin{array}{c}3 \\
(75)\end{array}$ & $\begin{array}{c}2 \\
(66.7)\end{array}$ \\
\hline Tigecycline & $\begin{array}{c}5 \\
(8.9)\end{array}$ & $\begin{array}{c}0 \\
(0)\end{array}$ & $\begin{array}{c}1 \\
(20)\end{array}$ & $\begin{array}{c}0 \\
(0)\end{array}$ & $\begin{array}{c}02 \\
(18.2)\end{array}$ & $\begin{array}{c}01 \\
(11.1)\end{array}$ & $\begin{array}{c}1 \\
(9.1)\end{array}$ & $\begin{array}{c}2 \\
(15.4)\end{array}$ & $\begin{array}{c}0 \\
(0)\end{array}$ & $\begin{array}{c}1 \\
(50)\end{array}$ & $\begin{array}{c}1 \\
(25)\end{array}$ & $\begin{array}{c}0 \\
(0)\end{array}$ \\
\hline Colistin & $\begin{array}{c}3 \\
(5.4)\end{array}$ & $\begin{array}{c}0 \\
(0)\end{array}$ & $\begin{array}{c}0 \\
(0)\end{array}$ & $\begin{array}{c}0 \\
(0)\end{array}$ & $\begin{array}{c}01 \\
(9.1)\end{array}$ & $\begin{array}{c}0 \\
(0)\end{array}$ & $\begin{array}{c}1 \\
(9.1)\end{array}$ & $\begin{array}{c}0 \\
(0)\end{array}$ & $\begin{array}{c}1 \\
(33.3)\end{array}$ & $\begin{array}{c}0 \\
(0)\end{array}$ & $\begin{array}{c}1 \\
(25)\end{array}$ & $\begin{array}{c}1 \\
(33.3)\end{array}$ \\
\hline $\begin{array}{c}\text { Trimethoprim } \\
\text { sulfamethoxazole }\end{array}$ & $\begin{array}{c}50 \\
(89.3)\end{array}$ & $\begin{array}{c}21 \\
(72.4)\end{array}$ & $\begin{array}{c}5 \\
(100)\end{array}$ & $\begin{array}{c}4 \\
(100)\end{array}$ & $\begin{array}{c}11 \\
(100)\end{array}$ & $\begin{array}{c}9 \\
(100)\end{array}$ & $\begin{array}{c}11 \\
(100)\end{array}$ & $\begin{array}{c}10 \\
(76.9)\end{array}$ & $\begin{array}{c}3 \\
(100)\end{array}$ & $\begin{array}{c}2 \\
(100)\end{array}$ & $\begin{array}{c}4 \\
(100)\end{array}$ & $\begin{array}{c}3 \\
(100)\end{array}$ \\
\hline Total $(\%)$ & $\begin{array}{c}56 \\
(100)\end{array}$ & $\begin{array}{c}29 \\
(100)\end{array}$ & $\begin{array}{c}05 \\
(100)\end{array}$ & $\begin{array}{c}04 \\
(100)\end{array}$ & $\begin{array}{c}11 \\
(100)\end{array}$ & $\begin{array}{c}09 \\
(100)\end{array}$ & $\begin{array}{c}11 \\
(100)\end{array}$ & $\begin{array}{c}13 \\
(100)\end{array}$ & $\begin{array}{c}3 \\
(100)\end{array}$ & $\begin{array}{c}2 \\
(100)\end{array}$ & $\begin{array}{c}4 \\
(100)\end{array}$ & $\begin{array}{c}3 \\
(100)\end{array}$ \\
\hline
\end{tabular}

CAUTI-Catheter Associated UTI; Non-CAUTI- Non-Catheter Associated UTI 
Table.4 Risk Factors Associated with BL and Non-BL Patients

\begin{tabular}{|c|c|c|}
\hline Risk factors & $\begin{array}{c}\mathbf{B L} \\
\mathbf{( N = 1 5 0 )}\end{array}$ & $\begin{array}{c}\text { Non-BL } \\
\mathbf{( N = 8 1 )}\end{array}$ \\
\hline Pregnancy & $45(30 \%)$ & $13(16 \%)$ \\
\hline Diabetes mellitus & $56(37.3 \%)$ & $6(7.4 \%)$ \\
\hline Renal disease/renal transplant & $68(45.3 \%)$ & $23(28.4 \%)$ \\
\hline Obstructive uropathy & $74(46.7 \%)$ & $16(19.8 \%)$ \\
\hline Obesity & $45(30 \%)$ & $8(9.8 \%)$ \\
\hline Prior antibiotic use within 3 months & $98(65.3 \%)$ & $32(39.5 \%)$ \\
\hline Long term hospital stay & $134(89.3 \%)$ & $41(50.6 \%)$ \\
\hline CAUTI & $125(83.3 \%)$ & $38(46.9 \%)$ \\
\hline ICU stay & $109(72.7 \%)$ & $29(35.8 \%)$ \\
\hline
\end{tabular}

Figure.1 Percentage of BL isolates from CAUTI patients

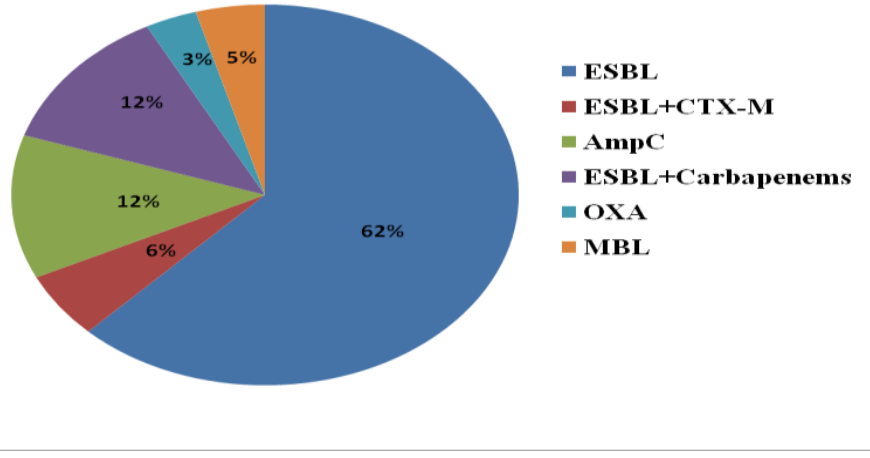

Figure.2 Percentage of BL isolates from Non-CAUTI patients

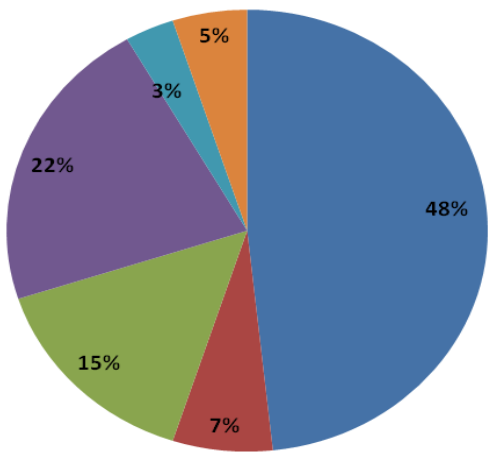

ESBL

ESBL+CTX-M

- AmpC

ESBL+Carbapenems

- OXA

MBL 
However, OXA and MBL are resistance to Imipenem and Meropenem. CTX-M, AmpC, OXA and MBL isolates are also resistance to Cefoxitin in both CAUTI patients and Non-CAUTI patients. But AmpC betalactamases were sensitive to $4^{\text {th }}$ generation cephalosporin, cefepime in both CAUTI patients and Non-CAUTI patients similar to the previous study conducted by Bush et al (2010). CTX-M and MBL showed resistance to both Cefoxitin and forth generation cephalosporin Cefipime in both CAUTI patients and Non-CAUTI patients. OXA and MBL are often resistance to Amikacin and Norfloxacin in both CAUTI patients and Non-CAUTI patients. OXA isolates showed even resistance to Gentamicin in both CAUTI patients and Non-CAUTI patients which was similar to what has been reported by other studies in developing countries (Hima-Lerible et al, 2003; Ndugulile et al, 2005; Ahmed et al, 2000). In this study, we found that the $\beta$-lactamases producing isolates were resistant to most of the antibiotics tested but Tigecycline and Colistin showed promising efficacy against all the Beta lactamases in both CAUTI patients and Non-CAUTI patients.

In conclusion, according to the present study the empirical use of antibiotics like Ampicillin and trimethoprimsulphamethoxazole, for UTI has not been proven efficient. The data regarding the prevalence of Beta Lactamase strains in CAUTI patients in Saudi Arabia is limited. Further studies regarding the BL producers in catheterized patients should be conducted in local hospitals in Saudi Arabia.

Catheterized patients should be screened regularly for the prevalence beta lactamase producers. While handling catheterized patients the healthcare staff should follow proper guidelines and infection control measures to avoid the spread of nosocomial infections. The Clinicians should follow local antibiotic policy for recommendations of alternative antibiotics to these patients.

\section{Acknowledgement}

We thank the Head of the laboratory and staff members of Microbiology department, Al-Dawadmi General Hospital for providing the necessary facilities to conduct the study. We also thank Shaqra University, MOHE, KSA for its support and encouragement.

\section{References}

Abdulrahman A. Kader, Angamuthu K. Kumar. Prevalence of extended spectrum $\beta$-lactamase among multidrug resistant gram-negative isolates from a general hospital in Saudi Arabia. Saudi Med J 2004; Vol. 25 (5): 570-574.

Ahmed AA, Osman H, Mansour AM, Musa $\mathrm{HA}$, Ahmed AB, Karrar $\mathrm{Z}$, et al. Antimicrobial agent resistance in bacterial isolates from patients with diarrhea and urinary tract infection in the Sudan. Am J Trop Med Hyg 2000; 63: 259-263.

Al-Agamy MH, Shibl AM, Tawfik AF, Prevalence and Molecular Characterization of ESBL producing Klebsiella pneumoniae in Riyadh, Saudi Arabia. Ann Saudi Med. 2009; 29:253257

Amreliwala.S, Durgad.S and A.Poojary,2015. Carbapenem sparing options for the treatment of ESBL and AmpC producing Enterobacteriaceae in hemodynamically stable patients an in vitro study, Int.J.Curr.Microbiol.App.Sci 4(2): 513521.

Arnoldo L, Migliavasca R, Regastin L, Raglio A, Pagani L, Nucleo E, Spalla M, Vailati F, Agodi A, Mosea A et al. Prevalence of urinary colonization by ESBL among catheterized inpatients in 
Italian long term care facilities. $B M C$ Infect Dis 2013, 13:124.

Azap O. K, Arslan H, Serefhanoqlu K, Colakoglu S, Erdogan H, Timurkaynak F,Senger S.S: Risk factors for ESBL positivity in uropathogenic Escherichia coli isolated from community-acquired urinary tract infections. Clin Microbiol Infect 2010, 16:147-151.

Babay H.A. Detection of extended $\beta$ lactamases in members of the family Enterobacteriaceae at a teaching hospital, Riyadh, Kingdom of Saudi Arabia. Saudi Med J 2002, 23: 186190.

Bandekar N, Vinodkumar CS, Basavarajappa KG, Prabhakar PJ, Nagaraj P. The beta lactamases mediated resistance amongst the gram negative bacilli in burn infections. Int J of Bio Res.2011; 2:76670.

Bilal NE, Gedebou M. Clinical and community strains of Klebsiella pneumoniae: multiple and increasing rates of antibiotic resistance in Abha, Saudi Arabia. Br J Biomed Sci 2000; 57: 185-191.

Bush K. Epidemiological expansion and clinical challenges of new lactamases. Ann Rev Microbiol. 2011; 65:455- 478.

Bush. K , G. A. Jacoby Updated Functional Classification of Beta Lactamases. Anti microb Agents Chemother 2010; 54(3): 969976.

Calbo E, Romani V, Xercavins M, Gomez L, Vidal C.G, Quintana S, Vila J, Garau J: Risk factors for community-onset urinary tract infections dueto Escherichia coli harbouring ESBLs. $J$ Antimicrob Chemother, 2006, 57:780783.

CLSI. Clinical and Laboratory Standards Institute (CLSI): Performance Standard for Antimicrobial Susceptibility Testing. 19th Informational supplement. CLSI document, 2012 M100-S22.

Conway, LJ and EL Larson, Guidelines to prevent catheter-associated urinary tract infection: 1980 to 2010. Heart \& Lung:
The Journal of Acute and Critical Care, 2012; 41(3): 271-283.

Hernandez JR, Pascual A, Canton R, Martinez-Martinez L; Grupo de Estudio de Infection Hospitalaria. GEIH. ESBL producing Escherichia coli and Klebsiella pneumoniae in spanish hospitals. Enferm Infecc Microbiol Clin 2003; 21: 77-82.

Hima-Lerible H, Ménard D, Talarmin A. Antimicrobial resistance among uropathogens that cause communityacquired urinary tract infections in Bangui, Central African Republic. $J$ Antimicrob Chemother 2003; 51: 192194.

Hooton TM, Bradley SF, Cardenas DD, Colgan R, Geerlings SE, Rice JC, Saint S, Schaeffer AJ, Tambyah PA, Tenke P, Nicolle LE: Diagnosis, prevention and treatment of CAUTI in adults; 2009. Clin Infect Dis 2010; 50:625-663.

Jaggi, $\mathrm{N}$ and $\mathrm{P}$ Sissodia, Multimodal Supervision Programme to Reduce CAUTI and Its Analysis to Enable Focus on Labour and Cost Effective Infection Control Measures in a Tertiary Care Hospital in India. Journal of Clinical and Diagnostic Research : JCDR, 2012; 6(8):1372-1376.

Karou SD, Iboudo DP, Nadembega WMC,Ameyapoh Y, Quermi D, Pignatelli $\mathrm{S}$ et al.Antibiotics resistane in urinary tract bacteriain Ouagadougou. Pak J Biol Sci 2009;12:712- 6.

Khalid Bindayna, Husam S. Khanfar, Abiola C. Senok, Giuseppe A. Botta, Predominance of CTX-M genotype among EEBL isolates in a tertiary hospital in Saudi Arabia, 2010, Saudi Med J, 31(8): 859-863.

Lo E, Nicolle LE, Coffin SE, Gould C, Maragakis L, Meddings J, Pegues DA, Pettis AM, Saint S, Yokoe DS: Strategies to prevent catheter-associated urinary tract infections in acute care hospitals: 2014 update. Infect Control Hosp Epidemiol 2014; 35:464-479. 
Loveena Oberoi, Nachhatarjit singh, PoonamSharma, Aruna Aggarwal. ESBL, MBL and Ampc $\beta$ Lactamases Producing Superbugs - Havoc in the Intensive Care Units of Punjab India. Journal of Clinical and Diagnostic Research. 2013;7(1): 70-73

Magill, SS., Hellinger, W., et al. "Prevalence of Healthcare-associated Infections in

Acute Care Facilities". Infection Control Hospital Epidemiology 2012; 33:283-91

Mathur P, Kapil A, Das B, Dhawan B. Prevalence of extended spectrum betalactamase producing Gram negative bacteria in tertiary care hospital. Indian J Med Res 2002; 115: 153- 157.

Mendonca N, Leitao J, Manageiro V, Ferreira E, Canica M. Spread of ESBL CTX-M producing Escherichia coli clinical isolates in community and nosocomial environments in Portugal. Antimicrob Agents Chemother 2007; 51: 1946-1955.

Mody L, Matieshwari S, Galecki A, Kauffman C.A, Bradley SF: Indwelling device use and antibiotic resistance in nursing homes: identifying a high-risk group. $J$ Am Geriatr Soc 2007; 55:1921-1926.

Moyo SJ, Aboud S, Kasubi M, LyamuyaEF, Maselle SY. Antimicrobial resistance among producers and non- producers of ESBL in urinary isolates at a tertiary hospital in Tanzania. BMC Research Notes 2010; 3:348-52.

Ndugulile F, Jureen R, Harthug S, Urassa W, Langeland N. ESBL among Gram- negative bacteria of nosocomial origin from intensive care unit of tertiary health facility inTanzania. BMC Infect Dis 2005; 5: 86.

Shafqat Qamar, 2015. Identification And Antibiotic Susceptibility Pattern Of ESBL Producing Enterobacteriaceae Among Patients With UTI. Ijbpas, 4(3): 1607-1615

Shah AA, Hasan F, Ahmed S, Hameed A. Extended-spectrum beta-lactamases in Enterobacteriaceae: related to age and gender. New Microbiol 2002; 25: 363366.

Sharma I, Paul D. Prevalence of community acquired urinary tract infections in Silchar Medical College, Assam, India and its antimicrobial susceptibility profile. Indian J Med Sci 2012; 66:1112.

Yamamichi F, Shigemura K, Matsumoto M, Nakano Y, Tanaka K, Arakawa S, Fujisawa M: Relationship between Urinary Tract Infection Categorization and Pathogens Antimicrobial Susceptibilities. Urol Int 2012, 88:198208.

Zilberberg MD, Shorr AF: Secular trends in gram-negative resistance among urinary tract infection hospitalizations in the United States, 2000-2009. Infect Control Hosp Epidemiol 2013; 34:940946.

\section{How to cite this article:}

Gouse Basha Sheik, Ahmed Sannat, Bader Al-Rowis, Sahar M. Jeddawy and Doaa M.Sherif. 2016. Prevalence, Risk Factors and Antimicrobial Susceptibility of Beta Lactamase Producing Enterobacteriacea in a Tertiary Care Centre of Saudi Arabia. Int.J.Curr.Microbiol.App.Sci. 5(2): 98-109. doi: http://dx.doi.org/10.20546/ijcmas.2016.502.011 